\title{
Erratum to: Military and veteran health behavior research and practice: challenges and opportunities
}

\author{
Jeffrey P. Haibach ${ }^{1}$ Michael Ann Haibach ${ }^{2}$ Katherine S. Hall,4 \\ Robin M. Masheb ${ }^{5,6} \cdot$ Melissa A. Little $^{7}$ Robyn L. Shepardson ${ }^{8,9}$ - Anne C. Dobmeyer ${ }^{10}$ • \\ Jennifer S. Funderburk ${ }^{8,9,11}$ - Christopher L. Hunter ${ }^{12}$ - Margaret Dundon ${ }^{13}$. \\ Leslie R. M. Hausmann ${ }^{14,15}$ - Stephen K. Trynosky ${ }^{16,17}$ • David E. Goodrich ${ }^{18}$. \\ Amy M. Kilbourne ${ }^{1,19} \cdot$ Sara J. Knight ${ }^{20,21} \cdot$ Gerald W. Talcott $^{7} \cdot$ Michael Goldstein $^{13}$
}

Published online: October 18, 2016

(C) Springer Science+Business Media New York 2016

\section{Erratum to: J Behav Med DOI 10.1007/s10865-016-9794-y}

The last name of Michael Ann Glotfelter is now changed to Michael Ann Haibach, and the affiliation for her should read as U.S. Air Force, Wright-Patterson Air Force Base, $\mathrm{OH}$, USA.

The original article was corrected.

The online version of the original article can be found under doi:10.1007/s10865-016-9794-y.

Jeffrey P. Haibach

Jeffrey.Haibach@va.gov

Michael Ann Haibach

Michael.Glotfelter.1@us.af.mil

Katherine S. Hall

Katherine.Hall@duke.edu

Robin M. Masheb

Robin.Masheb@yale.edu

Melissa A. Little

Mlitt118@uthsc.edu

Robyn L. Shepardson

Robyn.Shepardson@va.gov

Anne C. Dobmeyer

Anne.C.Dobmeyer.mil@mail.mil

Jennifer S. Funderburk

Jennifer.Funderburk@va.gov

Christopher L. Hunter

Christopher.L.Hunter16.mil@mail.mil

Margaret Dundon

Margaret.Dundon@va.gov
Leslie R. M. Hausmann

Leslie.Hausmann@va.gov

Stephen K. Trynosky

Steve.Trynosky@va.gov

David E. Goodrich

David.Goodrich2@va.gov

Amy M. Kilbourne

Amy.Kilbourne@va.gov

Sara J. Knight

Sara.Knight@va.gov

Gerald W. Talcott

Wtalcott@uthsc.edu

Michael Goldstein

Michael.Goldstein2@va.gov

1 Health Services Research and Development Service, U.S. Department of Veterans Affairs, 810 Vermont Avenue NW (10P9H), Washington, DC 20420, USA

2 U.S. Air Force, Wright-Patterson Air Force Base, OH, USA

3 Durham VA Medical Center, Durham, NC, USA 
4 Department of Medicine, Duke University, Durham, NC, USA

5 VA Connecticut Healthcare System, West Haven, CT, USA

6 Yale School of Medicine, New Haven, CT, USA

7 Center for Population Sciences, University of Tennessee Health Science Center, Memphis, TN, USA

8 VA Center for Integrated Healthcare, Syracuse VA Medical Center, Syracuse, NY, USA

9 Department of Psychology, Syracuse University, Syracuse, NY, USA

10 Defense Centers of Excellence for Psychological Health and Traumatic Brain Injury, Deployment Health Clinical Center, Bethesda, MD, USA

11 Department of Psychiatry, University of Rochester, Rochester, NY, USA

12 Patient-Centered Medical Home Branch, Clinical Support Division, Defense Health Agency, Washington, DC, USA

13 National Center for Health Promotion and Disease Prevention, U.S. Department of Veterans Affairs, Durham, NC, USA
14 Center for Health Equity Research and Promotion, VA Pittsburgh Healthcare System, Pittsburgh, PA, USA

15 Division of General Internal Medicine, Department of Medicine, University of Pittsburgh School of Medicine, Pittsburgh, PA, USA

16 Office of Academic Affiliations, U.S. Department of Veterans Affairs, Washington, DC, USA

17 Medical Service Corps, U.S. Army Reserve, Washington, DC, USA

18 Center for Clinical Management Research, VA Ann Arbor Healthcare System, Ann Arbor, MI, USA

19 Department of Psychiatry, University of Michigan Medical School, Ann Arbor, MI, USA

20 Birmingham VA Medical Center, Birmingham, AL, USA

21 Division of Preventive Medicine, University of Alabama at Birmingham, Birmingham, AL, USA 